\title{
Robust Grid Point-based Control Design for LPV Systems via Unified TP Transformation
}

\author{
Béla Takarics \\ Institute for Computer Science and Control \\ The Hungarian Academy of Sciences \\ Budapest, Hungary \\ Email:takarics@sztaki.mta.hu
}

\author{
Yeung Yam \\ Dept. of Mechanical and Automation Eng. \\ The Chinese University of Hong Kong \\ Shatin, N.T., Hong Kong \\ Email: yyam@mae.cuhk.edu.hk
}

\begin{abstract}
The paper considers a general approach for linear parameter varying (LPV) control. The approach is based on polytopic representation of the LPV system. First, a grid point based control design is applied for a set of linearized models of the LPV system. The design is based on linear time invariant (LTI) techniques. Such control design allows a larger flexibility in the controller structure than the conventional parallel distributed compensation (PDC) based polytopic control. The LPV controller is obtained by linear interpolation between the LTI controllers. The paper proposes linear matrix inequality (LMI) based convex optimization for robustness analysis of the resulting controller. The approach requires the plant and the controller to be defined by a common polytopic structure. It is proposed to obtain this common structure via unified TP model transformation. A simple numerical example shows the efficiency of the proposed control design approach.
\end{abstract}

Keywords-LPV control; robustness; TP model;

\section{INTRODUCTION}

A common approach to nonlinear control design is linear parameter-varying (LPV) control. The paper focuses on the polytopic LPV representation of nonlinear systems [1], [2]. The other two main LPV representations are linear fractional transformation (LFT) based LPV systems [3], [4], [5] and "grid-based" LPV systems [6], [7]. The polytopic representation of a nonlinear system can be obtained analytically [8] or numerically [9]. However, analytical derivations are often tedious or even impossible, particularly for more complex examples [9]. An efficient and tractable numerical method for obtaining the polytopic LPV representation is the tensor product (TP) model transformation as given in Section III-A [9]. The TP type polytopic form of a nonlinear system is obtained based on the higher-order singular value decomposition (HOSVD). The resulting TP model may exactly or approximately duplicate the original dynamics, depending on the singular values retained in the process. In turn, the singular values serve as a measure to trade off between the accuracy and complexity of the resulting model [9]. Recent directions in TP model control can be found in [10], [11], [12], [13], [14], [15], [16], [17], [18], [19].

The control design for TP type polytopic models is generally based on the parallel distributed compensation (PDC) framework [2] (Section II). The synthesis is done via linear matrix inequality (LMI) convex optimization for the linear time-invariant (LTI) vertex systems. The resulting LPV controller inherits the polytopic structure of the plant [2]. The flexibility of the polytopic control design is therefore limited by this fixed structure. Additionally, the flexibility is further limited as the synthesis step takes only the vertex systems into account.

The aim of the paper is to propose a control design strategy for LPV systems (Section IV) based on the work of [20], [21]. The main goal of the proposed design is to enable higher flexibility than general polytopic approaches. The flexibility of the control design can be enhanced by applying gain scheduling-like control design techniques as proposed in [20], [21]. The LPV system is linearized over a set of grid points and an LTI controller is designed for each for linearized model. This approach allows a large variety of LTI feedback design techniques to be applied. Additionally, the number of LTI systems can be arbitrarily high. Therefore, the control performance specifications can be tuned individually at each LTI system. Finally, the LPV controller is generated by linear interpolation between the LTI controllers. The drawback of such approach is that the resulting LTI controllers guaranty stability only in the vicinity of the LTI systems. Therefore, the second goal is to propose stability analysis via LMI feasibility tests or LMI-based convex optimization [2], [1] for the resulting LPV controller. The proposed approach requires the LPV system and the controller to have a common convex, polytopic representation. Such representation can be obtained by applying TP model transformation to the LPV system and to the controller. The controller designed with the proposed method however, has a polytopic structure that is in general different than the structure of the plant. A common polytopic structure can be obtained by applying the unified TP model transformation (Section III-B) upon which the LMI-based analysis can be executed [22].

It is assumed that the LPV model can have parametric 
uncertainties. Additional uncertainty may appear in case the TP model is only an approximation of the original LPV system. Therefore, it is proposed to verify robust stability of the resulting LPV controller based on [2] (Section III-C). The robust stability analysis is the main improvement over [20], [21]. The current paper treats state feedback design case only, but the results can be extended to output feedback design. A simple numerical example is given in Section $\mathrm{V}$ to demonstrate the effectiveness of the proposed method followed by the Conclusions.

\section{PROBLEM FORMULATION}

Consider the LPV system $G_{\rho}$ :

$$
\begin{aligned}
\dot{x} & =A(\rho(t)) x+B(\rho(t)) u \\
y & =C(\rho(t)) x+D(\rho(t)) u
\end{aligned}
$$

The signals are input $u(t) \in \mathbb{R}^{n_{u}}$, output $y(t) \in \mathbb{R}^{n_{y}}$ and state variable $x(t) \in \mathbb{R}^{n_{x}}$. Finally, $\rho(t) \in \mathbb{R}^{n_{\rho}}$ is a measurable exogenous parameter vector, called the scheduling parameter. $\rho$ is assumed to be a continuously differentiable function and the admissible trajectories are restricted based on physical considerations to a known compact subset $\mathcal{P} \subset$ $\mathbb{R}^{n_{\rho}}$. The rates of the parameter variation $\dot{\rho}$ are assumed to be bounded in some applications. The present paper investigates the unbounded rate case for simplicity. The dependence on time $t$ is suppressed in the remainder of the paper to shorten the notation. The system matrix $S(\rho)$ is given as

$$
S(\rho)=\left[\begin{array}{ll}
A(\rho) & B(\rho) \\
C(\rho) & D(\rho)
\end{array}\right]
$$

Note that $G_{\rho}$ is a quasi LPV (qLPV) system, belonging to the class of nonlinear systems, if the scheduling parameter $\rho$ contains elements of the state variable $x$.

A common way of LPV control is based on polytopic form and the PDC framework [2]. The finite element polytopic form of the LPV system $G_{\rho}$ can be given as

$$
S(\rho)=\sum_{r=1}^{R} w_{r}(\rho) S_{r}
$$

where $S_{r} \in \mathbb{R}^{\left(n_{x}+n_{u}\right) \times\left(n_{x}+n_{y}\right)}$ are the LTI vertex systems. The combination is defined by weighting functions $w_{r}(\rho) \in$ $\left[\begin{array}{ll}0 & 1\end{array}\right]$.

The controller is designed for the vertex LTI systems $S_{r}$ and the resulting LPV controller takes the same polytopic structure [2], [1], [23]. The control signal of the polytopic LPV control based on the PDC framework is generated in the following way

$$
u=-\sum_{r=1}^{R} w_{r}(\rho) F_{r} x
$$

where $F_{r} \in \mathbb{R}^{n_{x} \times n_{u}}$ are the LTI state feedback gains. A major limitation of this control approach is that the structure of the controller is determined by the structure of the plant. Additionally, the feedback gains are designed only for the LTI vertex systems. The goal is to propose an LPV control design method that can handle controllers with various structure providing additional flexibility in the control design. The key requirement for the proposed method is to provide robust stability analysis for the resulting controllers.

\section{TECHNICAL BACKGROUND}

This section reviews existing material on TP model transformation, unified TP model transformation and robustness analysis for polytopic LPV systems.

\section{A. TP Model Transformation}

The TP model transformation is a numerical method capable of transforming LPV systems to convex polytopic forms [9]. The TP model transformation was proposed as a methodology for system control design in [1]. The TP type polytopic form of the LPV system $G_{\rho}$ can be given as

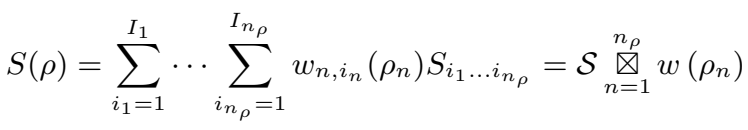
where
$\mathbb{R}^{I_{1} \times \cdots \times I_{n_{\rho}} \times\left(n_{x}+n_{u}\right) \times\left(n_{x}+n_{y}\right)}$ the has $n_{\rho}+2$ dimensions. $\mathcal{S}$ is constructed from the LTI vertex systems $S_{i_{1}, \ldots, i_{n_{\rho}}}$. The row vector $w_{n}\left(\rho_{n}\right)$ contains the one variable and continuous weighting functions $w_{n, i_{n}}\left(\rho_{n}\right)$ with $i_{n}=1 \ldots I_{n_{\rho}}$. The weighting functions $w_{n}\left(\rho_{n}\right)$ are determined based on singular matrices $U_{n}$ [9]. In order to have convex representation the weighting functions need to satisfy the following criteria:

$$
\forall n, i, \rho_{n}: w_{n, i}\left(\rho_{n}\right) \in[0,1] ; \quad \forall n, \rho_{n}: \sum_{i=1}^{I_{n}} w_{n, i}\left(\rho_{n}\right)=1
$$

Various types of convex representations can be constructed via TP model transformation. The current paper focuses on CNO type polytopic forms. A TP type convex model is CNO if the weighting functions satisfy the convexity criteria and largest value of all weighting functions is 1 or close to 1 .

It is important to note that not all LPV models belong to the class of TP models, i.e. they cannot be exactly reconstructed from finite number of vertex systems. Furthermore, occasionally the exact representation of LPV systems may lead to a complex polytopic structure that is not suitable for control design. In such cases an approximate TP model is more advantageous. Since HOSVD is the core of TP model transformation, an approximate model can be obtained by discarding small non-zero singular values. Assume the HOSVD of the LPV system $G_{\rho}$ is given and the $n$-mode rank of $S(\rho)$ is $R_{n}\left(1 \leq n \leq n_{\rho}\right)$ [9]. Let us define the approximated model as $\hat{S}(\rho)$. $\hat{S}(\rho)$ is obtained by discarding singular values $\sigma_{I_{n}^{\prime}+1}^{(n)}, \sigma_{I_{n}^{\prime}+2}^{(n)}, \ldots \sigma_{R_{n}}^{(n)}$ of tensor $\mathcal{S}$ for a given $I_{n}^{\prime}<R_{n}$. In this case the upper bound for the approximation can be given as

$$
\begin{aligned}
\frac{1}{\gamma_{T P}}= & \|\mathbf{S}(\mathbf{p}(t))-\hat{\mathbf{S}}(\mathbf{p}(t))\|^{2} \leq \\
& \sum_{i_{1}=I_{1+1}^{\prime}}^{R_{1}}\left(\sigma_{i_{1}}^{(1)}\right)^{2}+\cdots+\sum_{i_{n_{\rho}}=I_{n_{\rho}+1}^{\prime}}^{R_{n_{\rho}}}\left(\sigma_{i_{n_{\rho}}}^{\left(n_{\rho}\right)}\right)^{2}
\end{aligned}
$$




\section{B. Unified TP Model Transformation}

The unified TP model transformation was proposed in [22] as an extension of the TP model transformation. The unified TP model transformation is capable of transforming multiple LPV control system elements to a common polytopic structure. Consider the LPV system $G_{\rho}$ and assume an LPV state feedback controller $F(\rho)$ is available. The controller generates the control signal as

$$
u=-F(\rho) x
$$

Assume that the following vertex systems are obtained via TP model transformation:

$$
\mathcal{S} \underset{n=1}{n_{\rho}} U_{n}^{S}, \quad \mathcal{F} \underset{n=1}{n_{\rho}} U_{n}^{F}
$$

where $U_{n}^{S}$ and $U_{n}^{F}$ are the singular matrices, representing the discretized weighting functions (see [9] for more details about the steps of TP model transformation). Tensor $\mathcal{S}$ contains the LTI vertex systems of $G_{\rho}$ and tensor $\mathcal{F}$ contains the corresponding LTI feedback gains. The goal is to find a common $U_{n}$ for all components such that the weighting functions guarantee convex TP models:

$$
\mathcal{S}^{\prime} \underset{n=1}{n_{\rho}} U_{n}=\mathcal{S} \underset{n=1}{n_{\rho}} U_{n}^{S}, \quad \mathcal{F}^{\prime} \underset{n=1}{n_{\rho}} U_{n}=\mathcal{F} \underset{n=1}{n_{\rho}} U_{n}^{F}
$$

The discretized weighting functions $U_{n}^{S}$ and $U_{n}^{F}$ can be unified in the following way. Matrix $H$ is constructed as follows:

$$
H_{n}=\left[\begin{array}{ll}
U_{n}^{S} & U_{n}^{F}
\end{array}\right]
$$

In the next step, SVD is executed on $H$ with all the nonzero singular values kept. The discretized weighting functions are transformed to CNO form as

$$
H_{n}=U_{n}^{C N O} D_{n} V_{n}^{T}
$$

The product $D_{n} V_{n}^{T}$ is partitioned into matrices as

$$
D_{n} V_{n}^{T}=\left[\begin{array}{cc}
T_{n}^{S} & T_{n}^{F}
\end{array}\right]
$$

according to the dimension of $U_{n}^{S}$ and $U_{n}^{F}$ obtaining

$$
\left[\begin{array}{ll}
U_{n}^{S} & U_{n}^{F}
\end{array}\right]=U_{n}^{C N O}\left[\begin{array}{cc}
T_{n}^{S} & T_{n}^{F}
\end{array}\right]
$$

The common discretized weighting function can then be obtained in the following way

$$
\begin{aligned}
& \mathcal{S} \underset{n=1}{n_{\rho}} U_{n}^{S}=\mathcal{S} \underset{n=1}{n_{\rho}}\left(U_{n}^{C N O} T_{n}^{S}\right)= \\
& \left(\mathcal{S}{\underset{n=1}{n_{\rho}}}_{\bigotimes_{n}^{S}} T_{n=1}^{n_{\rho}} U_{n}^{C N O}=\mathcal{S}^{\prime}{\underset{n=1}{n_{\rho}}}_{n} U_{n}^{C N O}\right. \\
& \mathcal{F}{\underset{n=1}{n_{\rho}}}_{n} U_{n}^{F}=\mathcal{F} \underset{n=1}{n_{\rho}}\left(U_{n}^{C N O} T_{n}^{F}\right)= \\
& \left(\mathcal{F}{\underset{n=1}{n_{\rho}}}_{n} T_{n}^{F}\right){\underset{n=1}{n_{\rho}}}_{n}^{C N O}=\mathcal{F}^{\prime}{\underset{n=1}{n_{\rho}}}_{n}^{C N O} U_{n}^{C N O}
\end{aligned}
$$

\section{LMI-based Robustness Analysis}

Consider the uncertain LPV system $\hat{G}_{\rho}$ based on [2] with the following uncertainty structure

$$
\dot{x}=\left(A(\rho)+D_{a}(\rho) \Delta_{a} E_{a}(\rho)\right) x+\left(B(\rho)+D_{b}(\rho) \Delta_{b} E_{b}(\rho)\right) u
$$

where the uncertainty blocks satisfy

$$
\begin{gathered}
\left\|\Delta_{a}\right\| \leq \frac{1}{\gamma_{a}}, \quad \Delta_{a}=\Delta_{a}^{T} \\
\left\|\Delta_{b}\right\| \leq \frac{1}{\gamma_{b}}, \quad \Delta_{b}=\Delta_{b}^{T}
\end{gathered}
$$

The parameter dependent matrices $D_{a}(\rho), D_{b}(\rho), E_{a}(\rho)$ and $E_{b}(\rho)$ define the structure of the uncertainty. Assume that a common polytopic structure of the uncertain LPV model (16) and a corresponding LPV state-feedback controller of the form (8) is obtained. Then the following theorem holds for the state feedback control of $\hat{G}_{\rho}$ with controller $F(\rho)$ [2].

Theorem 1: The uncertain LPV system of (16) is stabilized via controller (8) if there exist a common positive definite matrix $P$ satisfying

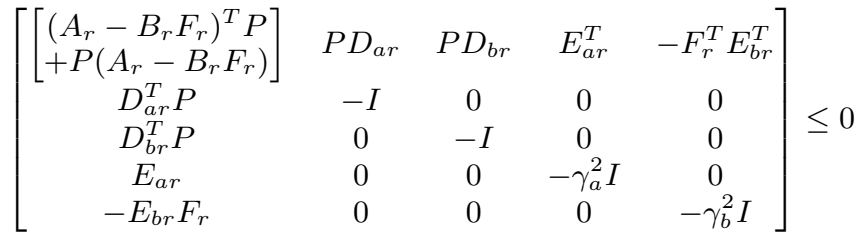

$$
\begin{aligned}
& {\left[\begin{array}{cccccc}
S_{r s} & T_{r s} & E_{a r}^{T} & -F_{s}^{T} E_{b r}^{T} & E_{a s}^{T} & -F_{r}^{T} E_{b s}^{T} \\
T_{r s}^{T} & -I & 0 & 0 & 0 & 0 \\
E_{a r} & 0 & -\gamma_{a}^{2} I & 0 & 0 & 0 \\
-E_{b r} F_{r} & 0 & 0 & -\gamma_{b}^{2} I & 0 & 0 \\
E_{a s} & 0 & 0 & 0 & -\gamma_{a}^{2} I & 0 \\
-E_{b s} F_{r} & 0 & 0 & 0 & 0 & -\gamma_{b}^{2} I
\end{array}\right] \leq 0}
\end{aligned}
$$

where the vertex feedback gains are given by $F_{r}(r=1 \ldots R$ and $R$ is the number of LTI vertex systems) for $r<s \leq R$, except the pairs $(r, s)$ such that $\forall \rho \in \mathbb{P}: w_{r}(\rho) w_{s}(\rho)=0$. Matrices $S_{r s}$ and $T_{r s}$ are defined as

$$
\begin{gathered}
S_{r s}=\left[\begin{array}{l}
\left(A_{r}-B_{r} F_{s}\right)^{T} P+P\left(A_{r}-B_{r} F_{s}\right)+ \\
+\left(A_{s}-B_{s} F_{r}\right)^{T} P+P\left(A_{s}-B_{s} F_{r}\right)
\end{array}\right] \\
T_{r s}=\left[\begin{array}{llll}
P D_{a r} & P D_{b r} & P D_{a s} & P D_{b s}
\end{array}\right]
\end{gathered}
$$

The proof of Theorem 1 is given in [2].

\section{PROPOSED METHOD}

The aim of this section is to give the technical details of the proposed control design strategy for LPV systems. The specific goals of the proposed methodology are

1) possibility to apply a large variety of LTI feedback design techniques;

2) flexible polytopic structure of the controller;

3) ability to tune the control performance based on the scheduling parameter $\rho$;

4) robustness analysis for the resulting LPV controller.

The proposed design is based on the idea given in [21], [20]. The key improvement is that the current method provides robustness analysis in addition to the control design. The proposed method has two main steps; the first is the grid point based control synthesis and the second is the robustness analysis of the resulting closed loop state feedback control. 
1) Grid Point-based Control Design: The main idea of the grid point-based control design is to synthesize LTI feedback controllers for a set of linearized models of the nominal LPV system $G_{\rho}$ [21], [20]. The first step is to obtain a set of linearized models of $G_{\rho}$ over a grid defined in $\mathcal{P}$. The linearized LTI systems are stored in tensor $\mathcal{S}^{\text {Grid }}$. The density of the grid should provide enough resolution and accuracy for the given problem. This also allows to tune the control performance specification based on the scheduling parameter $\rho$. Additionally, since LTI feedback gains are designed individually for each LTI system over the grid, the polytopic structure of the controller is not tied to the structure of the plant. The resulting LTI feedback gains are stored in tensor $\mathcal{F}^{\text {Grid }}$. Finally, the LPV controller $F(\rho)$ is generated by linear interpolation between the LTI feedback gains. The stability of the LTI controllers is guaranteed in the vicinity of the LTI systems. However, there is no guaranty that the LPV controller $F(\rho)$ is also stable.

2) Robustness Analysis: The second step is to verify the robust stability of the closed loop system of the LPV plant and the resulting LPV controller $F(\rho)$. The aim is to apply LMI-based convex optimization techniques for the stability analysis. Such approach can be applied for the TP type convex representation of the plant and the controller. It is assumed that the LPV system $G_{\rho}$ can have parametric uncertainty in the form of (16). An additional uncertainty is introduced in case an approximating TP model is used. Therefore, the stability of the resulting controller is verified for the uncertain LPV system $\hat{G}_{\rho}$ in addition to the nominal LPV system. The uncertainty blocks of $\hat{G}_{\rho}$ have the following structure

$$
\Delta_{a}=\Delta_{T P}+\Delta_{a_{\text {Model }}}, \quad \Delta_{b}=\Delta_{T P}+\Delta_{b_{\text {Model }}}
$$

where $\Delta_{T P}$ captures the approximation error of the TP model. $\Delta_{a_{\text {Model }}}$ and $\Delta_{b_{\text {Model }}}$ capture parametric uncertainties or unmodeled dynamics of the nominal LPV system $G_{\rho}$. An upper bound for $\Delta_{T P}$ can be given based on (7) as

$$
\left\|\Delta_{T P}\right\|=\frac{1}{\gamma_{T P}}
$$

The robustness analysis can be done in the following steps:

Step 1: The TP type polytopic forms of the LTI systems of $\mathcal{S}^{\text {Grid }}$ and the corresponding LTI controllers $\mathcal{F}^{\text {Grid }}$ are obtained via TP model transformation. The controller in general has a polytopic structure that is different than the structure of the plant. The TP model transformation results in the LTI vertex systems and gains with the discretized weighting functions as

$$
\mathcal{S}^{\text {Grid }}=\mathcal{S} \underset{n=1}{n_{\rho}} U_{n}^{S}, \quad \mathcal{F}^{\text {Grid }}=\mathcal{F}{\underset{n=1}{n_{\rho}}}_{n} U_{n}^{F}
$$

An upper bound on $\gamma_{T P}$ is also obtained in this step based on the discarded singular values for $\mathcal{S}^{\text {Grid }}$.

Step 2: The LTI vertex systems of $\mathcal{S}$ and the corresponding feedback gains of $\mathcal{F}$ are transformed to a common polytopic form based on the unified TP model transformation (Section III-B). The unified polytopic structure is given as

$$
\mathcal{S}^{\prime} \bigotimes_{n=1}^{n_{\rho}} U_{n}=\mathcal{S}{\underset{n=1}{n_{\rho}}}_{n_{n}} U_{n}^{S}, \quad \mathcal{F}^{\prime} \bigotimes_{n=1}^{n_{\rho}} U_{n}=\mathcal{F}{\underset{n=1}{n_{\rho}}}_{n} U_{n}^{F}
$$

where tensor $\mathcal{S}^{\prime}$ contains the LTI vertex systems, tensor $\mathcal{F}^{\prime}$ contains the corresponding state feedback gains and $U_{n}$ contains the discrete unified weighting functions for the common polytopic representation. The continuous weighting functions $w\left(\rho_{n}\right)$ are finally obtained from $U_{n}$. The resulting state feedback interconnection therefore takes the following structure

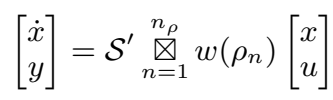

where the control signal $u$ is generated as

$$
u=-\mathcal{F}^{\prime} \underset{n=1}{n_{\rho}} w\left(\rho_{n}\right) x
$$

Step 3: The robustness analysis for the interconnection given by (24) and (25) can be done via convex optimization based on Theorem 1. The resulting $\gamma_{a}$ and $\gamma_{b}$ give an upper bound for $\left\|\Delta_{a}\right\|$ and $\left\|\Delta_{b}\right\|$. The upper bound on $\left\|\Delta_{T P}\right\|$ is given by $\gamma_{T P}$. $\gamma_{a_{M \text { odel }}}$ and $\gamma_{b_{M \text { odel }}}$ can then be calculated based on the following inequality

$$
\begin{aligned}
\left\|\Delta_{a}\right\| & \leq\left\|\Delta_{T P}\right\|+\left\|\Delta_{a_{\text {Model }}}\right\| \\
\left\|\Delta_{b}\right\| & \leq\left\|\Delta_{T P}\right\|+\left\|\Delta_{b_{\text {Model }}}\right\|
\end{aligned}
$$

It has to be noted, that in some cases it might not be possible to represent the controller $F(\rho)$ by finite element polytopic form, or the exact polytopic form can have undesirably high complexity. In such cases the TP approximation of the controller can be implemented for which the robust stability holds. The approximating capabilities of the TP model transformation ensure that the performance of the approximate controller remains close to the original performance criteria specified by the LTI feedback design.

\section{NUMERICAL EXAMPLE}

A simple numerical example is presented in order to show the efficiency of the proposed control design method. Consider a nonlinear mass-spring damper given in Figure 1 [8]. It is assumed that the nominal stiffness coefficient of the spring and the damping coefficient of the damper have nonlinearity as

$$
M \ddot{x}+g(x, \dot{x})+f(x)=u
$$

where $M$ is the mass and $u$ is the force. $f(x)$ is the nonlinear term of the spring and $g(x, \dot{x})$ is the nonlinear term of the damper.

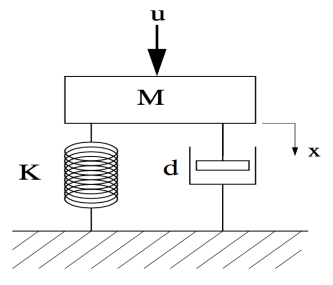

Figure 1. Mass-spring damper system

Assume that $g(x, \dot{x})=D\left(c_{1} x+c_{2} \dot{x}^{3}\right)$ and $f(x)=c_{3} x+$ $c_{4} x^{3}$. The parameters are set as follows: $M=1, D=1$, 
$c_{1}=c_{3}=0.01, c_{2}=0.1$ and $c_{4}=0.67$. The nominal mass-spring damper system can be given as

$$
\left[\begin{array}{c}
\dot{x} \\
\ddot{x}
\end{array}\right]=\left[\begin{array}{cc}
0 & 1 \\
-\left(0.02+0.67 x^{2}\right) & -0.1 \dot{x}^{2}
\end{array}\right]\left[\begin{array}{l}
x \\
\dot{x}
\end{array}\right]+\left[\begin{array}{l}
0 \\
1
\end{array}\right] u
$$

The region of interest is defined as $x \in\left[\begin{array}{ll}-1.5 & 1.5\end{array}\right]$ and $\dot{x} \in\left[\begin{array}{ll}-1.5 & 1.5\end{array}\right]$. The uncertain mass-spring damper system is defined based on the assumption that parameters $c_{1}=$ $c_{3}=0.01, c_{2}=0.1$ and $c_{4}=0.67$ can be uncertain.

\section{A. Grid Point-based Control Design}

First, the linearized models at a set of grid points within the region of interest are obtained. Consider the set of $N \times$ $M$ grid points as $\left[\begin{array}{ll}x_{1} & x_{2} \cdots x_{N}\end{array}\right] \times\left[\begin{array}{ll}\dot{x}_{1} & \dot{x}_{2} \cdots \dot{x}_{M}\end{array}\right]$, with $x_{1}=-1.5, x_{N}=1.5, \dot{x}_{1}=-1.5$ and $\dot{x}_{M}=1.5$. For values of $x$ and $\dot{x}$ close to $x_{i}$ and $\dot{x}_{j}$, the nonlinear plant can be approximated by the linear model

$$
\begin{aligned}
{\left[\begin{array}{c}
\dot{x} \\
\ddot{x}
\end{array}\right] } & =\left[\begin{array}{cc}
0 & 1 \\
-\left(0.02+0.67 x_{i}^{2}\right) & -0.1 \dot{x}_{j}^{2}
\end{array}\right]\left[\begin{array}{l}
x \\
\dot{x}
\end{array}\right]+\left[\begin{array}{l}
0 \\
1
\end{array}\right] u \\
& =A\left(x_{i}, \dot{x}_{j}\right)\left[\begin{array}{l}
x \\
\dot{x}
\end{array}\right]+B u
\end{aligned}
$$

The overall plant can then be approximated by a linear interpolation of the grid point models. The linearized models are stored in tensor $\mathcal{S}^{\text {Grid }}$ and the scheduling parameter can be defined as $\rho=\left[\begin{array}{ll}x & \dot{x}\end{array}\right]^{T}$.

Let the approach for control design in the present case be state feedback design with variable pole placement as given in [20]. The closed loop poles at the $(i, j)$ th grid point are set be equal to $\left(-2-0.1 \sqrt{x_{i}^{2}+\dot{x}_{j}^{2}},-2-0.1 \sqrt{x_{i}^{2}+\dot{x}_{j}^{2}}\right)$, i.e., a faster rate of decay is required if the states are further away from $x=0$ and $\dot{x}=0$. Therefore, the control law for each of the linearized model at the $(i, j)$ th grid point is given as $u=-F_{i j}\left[\begin{array}{ll}x & \dot{x}\end{array}\right]^{T}$. The LTI feedback gains are stored in tensor $\mathcal{F}^{\text {Grid }}$. The overall control for given values of $x$ and $\dot{x}$ is generated by linear interpolation.

\section{B. Robustness Analysis}

The goal is to verify the robust stability of the designed controller for the uncertain mass-spring damper system based on the proposed method.

Step 1: First, the TP type polytopic forms with the discrete weighting functions $U_{n}^{S}$ and $U_{n}^{F}$ of the nominal plant and controller are obtained. This is achieved by applying TP model transformation to tensors $\mathcal{S}^{\text {Grid }}$ and $\mathcal{F}^{\text {Grid }}$ individually. For the linearized models the nonzero singular values are: 208.6, 58.2 in the first dimension and 216.4 and 9.3 in the second dimension. Therefore, by keeping the 4 singular values the exact TP type polytopic representation of $\mathcal{S}^{\text {Grid }}$ is retained. The weighting functions for the plant are given in Figure 2.

The singular values for the controller in the first dimension are: $785.8,38.6,1.9,0.22,0.04,0.01, \ldots$ and in the second dimension: $786.6,13.9,1.45,0.2,0.04,0.01, \ldots$ The controller therefore cannot be described exactly by finite number of
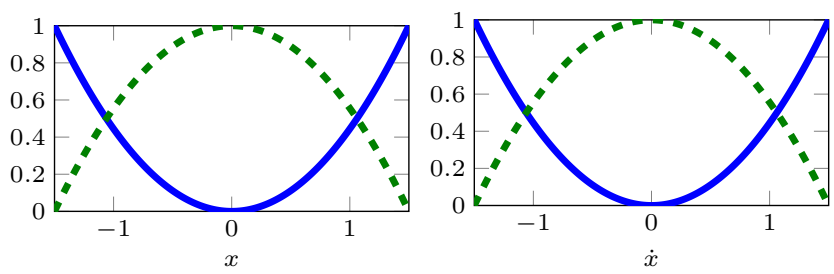

Figure 2. CNO type weighting functions for $\mathcal{S}^{\text {Grid }}$

elements. Keeping the first two nonzero singular values in each dimension ensures a good TP type approximation of the original controllers stored in $\mathcal{F}^{\text {Grid }}$. This ensures that the approximating controller has low complexity while the control performance remains similar to the performance of $\mathcal{F}^{\text {Grid }}$. The weighting functions are given in Figure 3.
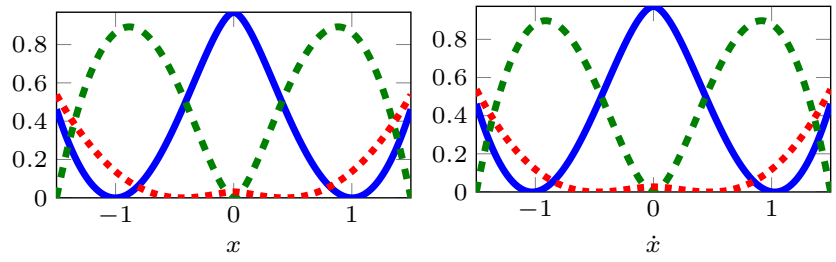

Figure 3. CNO type weighting functions for $\mathcal{F}^{\text {Grid }}$

Step 2: The next step is apply the unified TP model transformation to obtain a unified weighting structure for the LPV system and the controller. The resulting weighting functions are given in Figure 4.
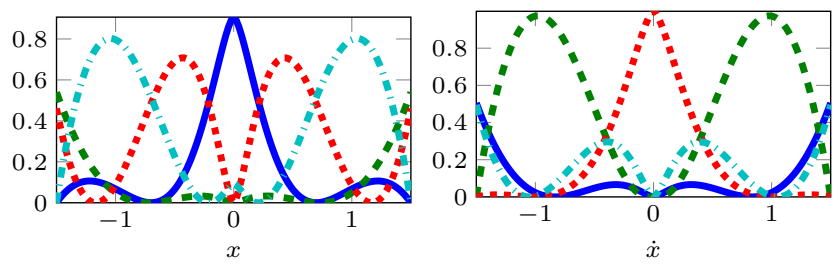

Figure 4. Unified $\mathrm{CNO}$ type weighting functions for $\mathcal{S}^{\prime}$ and $\mathcal{F}^{\prime}$

Step 3: The final step is the robustness analysis, which can be done via LMI convex optimization for the unified TP structure. First, the structure of the uncertainty needs to be defined. Recall, that in this case the TP form of the plant is an exact representation, since there were no nonzero singular values discarded. Therefore, $\Delta_{T P}=0$. The structure of the uncertainties in parameters $c_{1}=c_{3}=0.01, c_{2}=0.1$ and $c_{4}=0.67$ are captured by

$$
D_{a}=\left[\begin{array}{cc}
0 & 0 \\
-1 / M & -1 / M
\end{array}\right], \quad E_{a}=\left[\begin{array}{ll}
1 & 0 \\
0 & 1
\end{array}\right]
$$

The proposed robustness analysis for this uncertain LPV model results in $\gamma_{a}=0.445$. Therefore, it is shown that the approximate TP type polytopic form for the designed controller robustly stabilizes the uncertain qLPV mass-spring damper system in addition to the nominal model. $\gamma_{a}=0.445$ gives an upper bound for the allowable uncertainty. 


\section{CONCLUSIONS}

The paper proposed an approach for LPV control design based on grid point feedback design. The grid point approach allows high flexibility in the design, but guaranties stability only in the vicinity of the LTI systems. LMI based robust stability analysis is proposed to verify the robust stability of the resulting state feedback interconnection. The stability is verified based on convex polytopic representation of the LPV system and the designed controller. The paper proposed unified TP model transformation to obtain the common polytopic form of the controller and the plant. The robust stability analysis can be evaluated for the resulting common polytopic form. The benefits of the proposed method are shown by a simple numerical example. The future plan is to extend the proposed control design to output feedback.

\section{ACKNOWLEDGMENT}

This research described in this paper is partially supported by a General Research Fund from the Research Grant Council of the Hong Kong Special Administration Region, China (Ref. No. 418212).

\section{REFERENCES}

[1] P. Baranyi, "TP model transformation as a way to LMIbased controller design," IEEE Transactions on Industrial Electronics, vol. 51, no. 2, pp. 387-400, 2004.

[2] K. Tanaka and H. Wang, Fuzzy control systems design and analysis: a linear matrix inequality approach. WileyInterscience, 2001.

[3] A. Packard, "Gain scheduling via linear fractional transformations," Systems \& Control Letters, vol. 22, no. 2, pp. 79-92, Feb. 1994.

[4] P. Apkarian and P. Gahinet, "A convex characterization of gain-scheduled $H_{\infty}$ controllers," IEEE Transactions on Automatic Control, vol. 40, no. 5, pp. 853-864, May 1995.

[5] J. Veenman and C. W. Scherer, "Stability analysis with integral quadratic constraints: A dissipativity based proof." IEEE, Dec. 2013, pp. 3770-3775.

[6] F. Wu, "Control of linear parameter varying systems," Ph.D. dissertation, Univ. California, Berkeley, 1995.

[7] F. Wu, X. Yang, A. Packard, and G. Becker, "Induced $\mathcal{L}_{2}$-norm control for LPV systems with bounded parameter variation rates," Int. J. Robust Nonlinear Control, vol. 6, pp. 2379-2383, 1996.

[8] K. Tanaka, T. Ikeda, and H. Wang, "Robust stabilization of a class of uncertain nonlinear systems via fuzzy control: quadratic stabilizability, H/sup / control theory, and linear matrix inequalities," IEEE Transactions on Fuzzy Systems, vol. 4, no. 1, pp. 1-13, Feb. 1996.

[9] P. Baranyi, Y. Yam, and P. Vrlaki, Tensor product model transformation in polytopic model-based control, ser. Automation and control engineering series. Boca Raton: CRC Press, 2014.
[10] S. Kuntanapreeda, "Tensor product model transformation based control and synchronization of a class of fractionalorder chaotic systems," Asian Journal of Control, vol. 17, no. 2, pp. 371-380, Mar. 2015.

[11] S. Chumalee and J. F. Whidborne, "Gain-scheduled $H_{\infty}$ control for tensor product type polytopic plants," Asian Journal of Control, vol. 17, no. 2, pp. 417-431, Mar. 2015.

[12] A. Hajiloo and W. F. Xie, "The stochastic robust model predictive control of Shimmy vibration in aircraft landing gears," Asian Journal of Control, vol. 17, no. 2, pp. 476-485, Mar. 2015.

[13] P. Baranyi, "TP model transformation as a manipulation tool for qLPV analysis and design," Asian Journal of Control, vol. 17, no. 2, pp. 497-507, Mar. 2015.

[14] J. Pan and L. Lu, "TP model transformation via sequentially truncated higher-order singular value decomposition," Asian Journal of Control, vol. 17, no. 2, pp. 467-475, Mar. 2015.

[15] T. T. Wang, W. F. Xie, G. D. Liu, and Y. M. Zhao, "QuasiMin-Max model predictive control for image-based visual servoing with tensor product model transformation," Asian Journal of Control, vol. 17, no. 2, pp. 402-416, Mar. 2015.

[16] J. Kuti, P. Galambos, and . Mikls, "Output feedback control of a dual-excenter vibration actuator via qLPV model and TP model transformation," Asian Journal of Control, vol. 17, no. 2, pp. 432-442, Mar. 2015.

[17] J. Matusko, S. Iles, F. Kolonic, and V. Lesic, "Control of 3d tower crane based on tensor product model transformation with neural friction compensation," Asian Journal of Control, vol. 17, no. 2, pp. 443-458, Mar. 2015.

[18] R.-E. Precup, E. M. Petriu, M.-B. Rdac, S. Preitl, L.-O. Fedorovici, and C.-A. Drago, "Cascade control system-based cost effective combination of tensor product model transformation and fuzzy control," Asian Journal of Control, vol. 17, no. 2, pp. 381-391, Mar. 2015.

[19] B. Takarics and P. Baranyi, "Friction compensation in TP model form - aeroelastic wing as an example system," Acta Polytechnica Hungarica, vol. 12, no. 4, pp. 127-145, 2015.

[20] Yeung Yam and P. Baranyi, "Stabilizing controller by local linearization and SVD consolidation," vol. 2. IEEE, 2003, pp. 1482-1487.

[21] Yeung Yam and Wai Man Lee, "Fuzzy controller/observer via grid point design and SVD consolidation.” IEEE, 2003, pp. 5526-5531.

[22] P. Baranyi and B. Takarics, "Aeroelastic wing section control via relaxed tensor product model transformation framework," Journal of Guidance, Control, and Dynamics, vol. 37, no. 5, pp. 1671-1678, 2014.

[23] B. Takarics and P. Baranyi, "Tensor-product-model-based control of a three degrees-of-freedom aeroelastic model," Journal of Guidance, Control, and Dynamics, vol. 36, no. 5, pp. 1527-1533, 2013. 